\title{
Optical quality analysis system assessed optical quality in Chinese Primary Open Angle Glaucoma patients and its correlations with psychological disturbances and vision related quality of life
}

\author{
Yang Zhang \\ PUMCH https://orcid.org/0000-0002-2844-0572 \\ Ailing Bian \\ pumch \\ Qijun Hang \\ University of Pittsburgh \\ Qi Zhou ( $\sim$ zqhy_107@126.com) \\ $\mathrm{PUMCH}$
}

\section{Research article}

Keywords: Glaucoma, 25-Item National Eye Institute Visual Function, Quality of life, Optical quality analysis system, Hospital Anxiety and Depression Scale

Posted Date: November 22nd, 2019

DOI: https://doi.org/10.21203/rs.2.17702/v1

License: (a) (i) This work is licensed under a Creative Commons Attribution 4.0 International License.

Read Full License 


\section{Abstract}

Purpose To investigate if optical quality assessed by Optical Quality Analysis System(OQAS)is a new factor for psychological disturbance and vision related quality of life $(\mathrm{Q} o L)$ in Chinese patients with primary open angle glaucoma (POAG).

Methods Cross-sectional study. OQAS, automated static perimetry, the Hospital Anxiety and Depression Scale (HADS) Questionnaire and the 25-item National Eye Institute Visual Function Questionnaire (NEI VFQ-25) were used to assess optical quality, vision field, psychological health and QoL in 64 POAG patients. Correlation between the OQAS parameters and QoL or psychological health was analyzed by linear regression model.

Results OQAS parameters, the modulation transfer function cut off frequency (MTF cutoff) and the mean OQAS values (mean OV), were significantly related to mean deviation (MD). Statistically significant correlation was also found between MTF cutoff, the mean OV and QoL in more than half of the NEI VFQ25 subscale scores and the composite. The correlation coefficients between OQAS parameters and QoL were similar to those between MD and QoL in most of the subscale scores. HADS-Depression and HADSAnxiety scores significantly correlated with MD in the worse eye, the composite and 3subscales of NEI VFQ-25, general health, social function and mental health, but uncorrelated with optical quality parameters measured by OQAS.

Conclusions OQAS assessed optic quality is negatively related to MD and is a valid indicator for vision related QoL, but not for psychological health in our study group. OQAS might be an alternative or supplement to perimeter in POAG patients.

\section{Introduction}

Glaucoma is a leading cause of irreversible vision loss and permanent damage of visual function, which will further adversely influence the quality of life区QoL『and impose a psychological burden on patients[1, 2]. And the psychological disorder will in turn negatively affect their quality of life. The goal of glaucoma treatment is to maintain visual function and vision related quality of life of the patients, and to improve their mental health. Understanding of influencing factors to QoL and psychological health will ultimately benefit glaucoma treatment.

Glaucoma patients have a greater likelihood to be in the mood of anxiety and depression, the two common forms of psychological disturbance[3]. Up to date, several factors have been reported to be associated with psychological disturbance in patients with glaucoma, such as younger age, being female and having a moderate or heavy economic burden $[4,5]$.

Vision field is the most commonly used functional test for glaucoma diagnosis and follow up. Mean deviation (MD) of the vision field has been found to be a factor for QoL and psychological health. 
However vision field test is more subjective and depends highly on the cooperation of the subjects. Thus the result is not always reproducible. A good surrogate biomarker is keenly needed in the clinic.

In recent years, Optical Quality Analysis System (OQAS), a new instrument based on recording images from a point source object after reflection on the retina and a double pass through the ocular media, was developed and demonstrated to be a useful tool for comprehensively evaluating the objective optical quality of the eye[6]. It is recognized that OQAS has the advantages of easy operation, time saving, being objective and has good repeatability. However the association between QoL, psychological health outcomes and optical quality parameters measured by OQAS in patients with glaucoma has not been established. To find out if OQAS can effectively demonstrate the optic quality of glaucoma patients and if it is also a factor for QoL and psychological disturbances as MD, we used OQAS to assess the optical quality and evaluated its relationship to QoL and psychological status in primary open angle glaucoma patients.

\section{Materials And Methods}

\subsection{Study Participants and Protocol}

This study was approved by the Institutional Review Board of Peking Union Medical College Hospital and conformed to the tenets of the Declaration of Helsinki. Informed consent was obtained from all subjects before entry into this study.

From January 1, 2017 to June 31, 2017, 64 glaucoma patients at ophthalmology department, Peking Union Medical College Hospital, were included in the study.

The inclusion criteria were adult patients (18 years old and above) with primary open angle glaucoma (POAG) based on glaucomatous excavation of the optic nerve head, and reproducible VF damage detected by automated static perimetry in one or both eyes. The exclusion criteria were as follows: (1) secondary glaucoma; (2) any other coexisting ocular condition that could impair visual function and the test results(e.g., clinically significant cataract, macular degeneration or any other ophthalmic condition); (3) history of incisional ocular surgery or laser treatment; and (4) disability in a VF test and/or questionnaire test due to cognitive impairment; (5) any other ocular or systemic disease that could affect the optic nerve or VF; (6) persons with systemic or social conditions that would cause emotional changes. (7) persons with cognitive problems that would exclude the possibility of questionnaire process.

Patients underwent a comprehensive ophthalmic examination, including best-corrected visual acuity (BCVA), slit lamp biomicroscopy, dilated fundus examination, intraocular pressure measurement using Goldmann tonometry, gonioscopy, stereoscopic optic disc photography (Canon, CR-1 Mark II), VF examination, and retinal nerve fiber layer assessment by optical coherence tomography (Stratus OCT, Carl Zeiss Meditec, CA). 
VF examinations were performed with the Octopus 101 perimeter (Haag-Streit, Inc, Koeniz, Switzerland) tG2 program with Tendency Oriented Perimetry strategy. Visual fields were reviewed and excluded in the presence of artifacts such as eyelid or rim artifacts, fatigue effects, inattention, inappropriate fixation, or abnormalities that could indicate diseases other than glaucoma. MD were used to assess the severity of VF loss. The eye with a higher score was defined as the better eye.

QoL was measured with the Chinese version of NEI VFQ-25. This questionnaire measures the influence of visual disability and visual symptoms on general health domains such as emotional well-being and social functioning, and daily visual function related task-oriented domains. It provides scores on 12 scales and a composite. The composite is the average score of the 12 subscales, including general health, general vision, ocular pain, near vision, distant vision, social functioning, mental health, role limitations, dependency, driving, color vision, and peripheral vision. The standard algorithm was used to calculate the scale scores, ranged from 0 to 100. A higher score represents better visual functioning and well-being.

Psychological symptoms of anxiety and depression in POAG patients were assessed using a Chinese version of Hospital Anxiety and Depression Scale (HADS). HADS is a 14-item scale with seven items for HADS-anxiety (HADS-A) and seven for HADS-depression (HADS-D) subscale. Scoring for each item ranges from zero to three, with three denoting highest anxiety or depression level. Higher scores indicate a higher level of depression and anxiety. A total subscale score over 8 out of 21 denotes considerable symptoms of anxiety or depression.

The subjects were requested to answer the questionnaire by themselves. The research staff explained the questionnaire to them and provided assistance when required. For participants who could not read due to poor eyesight, a research staff would read the questionnaire to them in a neutral and uniform manner and record their choices.

Optical quality parameters, such as the modulation transfer function cut off frequency (MTF cutoff), objective scatter index (OSI) and the mean OQAS values (OV, the mean of OV100\%, OV20\%, and OV9\%) were measured using the OQAS II (Visiometrics, Terrassa, Spain). Standard test protocol was followed and was described in detail in previous study[7].The room illumination was kept low (approximately 25 lux) during testing. The manifest refractive error of the subjects was fully corrected during these measurements; the spherical error (up to $-8.00 \mathrm{D}$ ) was automatically corrected by the double-pass system, and the residual spherical error (over $-8.00 \mathrm{D}$ ) as well as the cylindrical error was corrected with an external lens, because the uncorrected refractive error directly affects the optical outcome of the system. Since these optical quality parameters are known to be affected by pupil diameter, we confirmed that the pupil diameter was more than $4.0 \mathrm{~mm}$ in all eyes during the test.

\subsection{Statistical Analysis}


Mean and standard deviation (SD) were used to analyze variables. A single linear regression analysis was conducted to assess the relationship between the composite and 12 subscale scores of the NEIVFQ-25, HADS scores and optical quality parameters measured by OQAS. The level of statistical significance was set at 0.05 . All statistical analyses were carried out using statistical analysis software, SPSS version 14 for Windows (SPSS, Chicago, IL, USA).

\section{Results}

There were $42(65.6 \%)$ men and 22(34.4\%)women enrolled in this study, with a mean age of $56.6 \pm 15.7$ years, ranging from 27 to 79 years.

\subsection{OQAS assessed optic quality and vision field}

MTF cutoff $(-0.551, P<0.001)$ and mean OV $(-0.558, P<0.001)$ are both negatively correlated significantly with MD in total, better and worse eyes. MD, MTF cutoff, mean OV, OSI of the better eye and worse eye are displayed in Table1.

\subsection{OQAS assessed optic quality, vison field and NEI VFQ- 25}

The scores of the NEI VFQ-25 are shown in Fig. 1. The lowest score was seen in general health (32.03), followed by general vision, eye pain and mental health, the highest score was seen in color vision (97.66) followed by role limitations and distance vision.

A monotonic trend was observed between MD, MTF cutoff, mean OV and QoL in more than half of NEI VFQ-25 subscale scores. Linear regression coefficients for the association between OQAS index, MD and NEIVFQ-25 scores are shown in Table 2 for both the better and worse eye.

The NEI VFQ-25 results correlated closely with MD, the value of correlation coefficients between the NEI VFQ-25 and better eye VF were slightly higher than those between the worse eye in the NEI VFQ-25 composite score and 6/12 NEI VFQ-25 subscales, including general vision, near vision, distance vision, role limitation, driving and peripheral vision. Patients with severer VF loss had the greatest difficulty with distance vision and peripheral vision.

Statistically significant correlation was found between MTF cutoff and composite of the NEI VFQ-25 results, as well as 6/12 NEI VFQ-25 subscales, including general health, general vision, near vision, distance vision, dependency and peripheral vision in worse eyes. Statistically significant correlation was also found in almost the same subscales in better eyes, except general health and composite. Patients with worse MTF cutoff had the greatest difficulty with peripheral vision. The correlation coefficients between the mean OV and NEI VFQ-25 results were slightly lower than those between MTF cutoff and 
NEI VFQ-25 results. The correlation coefficients between mean OV in the better eye and NEI VFQ-25 results were significant in 6 subscales ranged from 0.258 to 0.406 and in the worse eye in composite and 6 subscales from 0.253 to 0.426 .

MD, MTF cutoff, mean OV all demonstrated high correlations in peripheral vision, general vision, near vision and distance vision subscales in the NEI VFQ-25 results. And the correlation coefficients between MTF cutoff, mean OV, MD and QoL follow similar trend. (Fig. 2)

No statistically significant correlation was found between OSI and composite of the NEI VFQ-25 as well as any other subscales.

\subsection{OQAS optic quality, vision field and psychological health.}

The prevalence of depression and anxiety symptoms was $15.6 \%$ and $28.1 \%$ respectively among POAG patients in this study. Mean HADS-Depression and HADS-Anxiety scores were $3.87 \pm 3.26$ and $5.8 \pm 3.34$.

HADS-Depression and HADS-Anxiety scores significantly correlated with MD in worse eye $(0.596, P=$ $0.009 ; 0.705, P=0.023)$, the composite $(-0.370, P=0.003 ;-0.269, P=0.032)$ and 3 subscales, general health $(-0.420, P=0.001 ;-0.466, P<0.001)$, social function $(-0.474, P<0.001 ;-0.379, P=0.002)$ and mental health $(-0.644, P<0.001 ;-0.537, P<0.001)$, but uncorrelated with optical quality parameters measured by OQAS.

\section{Discussion}

Glaucoma is a life-long chronic psychosomatic vision threatening disease. Optic quality and its relationship with vision related QoL and mental health is key for glaucoma treatment and long-term prognosis.

OQAS indexes, including MTF cutoff and OV, are new optical quality parameters. MTF is the ratio of contrast between the retinal image and the original scene. It represents the loss of contrast produced by the eye's optics as a function of spatial frequency. The OV100\%, OV $20 \%$, and OV9\% are normalized values of three spatial frequencies and describe the optical quality of the eye for three contrast conditions, commonly used in ophthalmic practice: $100 \% \otimes 20 \%$ and $9 \%$, respectively. And mean OV, the mean of OV100\% OV $20 \%$ and OV9\%, is sometime used to represent the overall optic quality of human eyes. The higher the value of the MTF cutoff and mean OVs, the better the optical quality. It is now widely used in refractive surgery, dry eyes and cataract grading. However, Wang et al found eyes with ocular hypertension had lower MTF cutoff and OVs compared with control eyes, which indicated that the contrast sensitivity in OHT eyes is not as good as healthy ones. They also found the contrast sensitivity changes in OHT eyes detected by OQAS appeared earlier than visual field changes[8]. In this study, we found MTF cutoff and mean OV were both negatively correlated significantly with MD in the better, worse 
and all eyes. This shows OQAS can also detect changes in glaucoma eyes and OQAS indexes, MTF cutoff and mean OV might be able to be a surrogate for MD. Several studies have shown that OQAS assessed ocular quality is affected by retina change[9, 10]. We speculate that the variation of OQAS values in glaucoma eyes might be caused by glaucomatous retinal changes, such as changes in the thickness of RNFL, RGC, or even blood flow. Further studies are needed to elucidate the exact mechanism.

In this study, we chose the NEI VFQ-25 to demonstrate the impact of glaucomatous visual function impairment of POAG patients. It measures the impact of visual disability and visual symptoms on general health, social functioning, and task-oriented daily visual functioning. It has been used and shown to be internally consistent, reproducible and responsive in glaucoma patients[11, 12], although it was not specifically designed for patients with glaucoma. It has already been used in investigating the relationship between QoL and influencing factors, such as visual field (VF) loss and glaucomatous retinal nerve fiber layer (RNFL) loss in glaucoma patients[13, 14]. Gutierrez et al found a steady linear decline between VF loss and QoL in glaucoma patients, suggesting that prevention of early VF loss may be critical for maintaining patients'QoL[15]. Consistent with previous research[13, 16, 17], we found significant relationship between VF defects and impaired QoL by NEI VFQ-25 in patients with glaucoma.

Our study demonstrated that the correlation coefficients between MTF cutoff, mean OV and QoL were similar to those between MD and QoL in more than half subscales. It means OQAS can provide additional information of vision related QoL in glaucoma patients. However, just like MD, OQAS indexes don't have a particularly close connection with some NEI VFQ-25 subscales. This means, just like visual field damage, glaucomatous OQAS changes did not affect all the QoL in the same degree and bilateral function might compensate single eye dysfunction in some subscales. There is also a possibility that patients might adapt to the slow progressive damage thus alleviate the impact of visual loss on activities of daily life.

Different studies have drawn different conclusions on whether the better or the worse functioning eyes is related closer to QoL. Although Gutierrez P et al. concluded that the better eye was more significantly related to QoL[15], Jampel et al. reported no significant difference between the two eyes[18]. In the current study, for the composite, we found the correlation coefficients between MTF cutoff, mean OV and QoL was more significant in the worse eyes than in the better eyes, however, the overall trend was similar for subscales between the two eyes. No matter what the conclusion would be, we should pay equal attention to the better and worse eyes in the clinic.

The Hospital Anxiety and Depression Scale (HADS) is a self-assessment questionnaire that has been found to be a reliable instrument for detecting level of anxiety and depression in glaucoma patients. The rates of depression and anxiety symptoms found in our study was $15.6 \%$ and $28.1 \%$ respectively. These were higher than normal population and close to the reported prevalence of $16.40 \%$ and $22.92 \%$ in Chinese glaucoma patients[5]. This echoed that glaucoma did affect patients' mental health. We found in our study that HADS-Depression and HADS-Anxiety scores significantly correlated with MD in worse eye and OoL scores, but uncorrelated with optical quality parameters measured by OQAS. Psychological status measurement is a patient-reported outcome, it tends to be more reflective of subjective measures 
than objective measures. These might imply that OQAS parameters are more objective than MD of visual field. It suggests that, ophthalmologists in clinical practice should interpret the results of visual field in consideration of the accompanying anxiety and depression symptoms in patients who appear to progress in VF but do well in OQAS measures.

OSI is another important index of OQAS. In the current study, we did not find good correlation between OSI and MD. Neither statistically significant correlation was found between OSI and composite of the NEI VFQ-25 as well as any other subscales. The possible explain might be that OSI is a parameter sensitive to lens opacity, while in our study, any abnormality in lens were excluded. MD is also a value after correction of the impact of cataract. However, OSI may play a role in glaucoma patients with cataract. In conjunction with MTF cutoff and mean OV, OQAS can provide important information on the prognosis of cataract surgery on glaucoma cataract patients and monitor glaucoma progress without the impact of cataract.

There are several limitations in our study. The sample size is too small, and we don't make stratification of severity by VF and optic quality, this may limit its ability to draw a solid conclusion between OQAS and QoL and mental health. We did not collect and analyze the social and economic factors of the patients, which are known factors affecting mental health. Another limitation of our study is the lack of longitudinal data on the association between OQAS indexes and QoL and mental health. More subjects will be enrolled and longer follow up will be planned.

In summary, OQAS indexes might be more subjective than vision field. It correlated well with vision-related quality of life in Chinese patients with primary open angle glaucoma, they are valid indicators for the degree of self-reported disability associated with glaucoma and may add to vision field test in glaucoma management.

\section{Abbreviations}

BCVA: best-corrected visual acuity, HADS: Hospital Anxiety and Depression Scale, MD: mean deviation, MTF cutoff: modulation transfer function cut off frequency, NEI VFQ-25: 25-item National Eye Institute Visual Function Questionnaire, OQAS: Optical Quality Analysis System, OSI: objective scatter index, OV: OQAS values, POAG: rimary open angle glaucoma, QoL: quality of life, VF: visial field

\section{Declarations}

- Ethics approval and consent to participate: This study was approved by the Institutional Review Board of Peking Union Medical College Hospital and conformed to the tenets of the Declaration of Helsinki. Written informed consent was obtained from all subjects after an explanation of the nature of the study before entry into this study.

- Consent for publication: Not applicable. 
- Availability of data and materials: The datasets used and analyzed during the current study are available from the corresponding author on reasonable request.

- Competing interests: The authors declare that they have no competing interests.

- Funding: None.

- Authors' contributions: YZ wrote the main manuscript text and prepared all figures. QZ oversaw the project and assisted with the writing of the manuscript. ALB performed ophthalmic examinations. All authors reviewed the manuscript.

- Acknowledgements: Not applicable.

\section{References}

[1] Weinreb RN, Aung T, Medeiros FA. The pathophysiology and treatment of glaucoma: a review. JAMA. 2014;311:1901-11.

[2] Jampel HD, Frick KD, Janz NK, Wren PA, Musch DC, Rimal R, et al. Depression and mood indicators in newly diagnosed glaucoma patients. Am J Ophthalmol. 2007;144:238-44.

[3] Wang SY, Singh K, Lin SC. Prevalence and predictors of depression among participants with glaucoma in a nationally representative population sample. Am J Ophthalmol. 2012;154:436-44 e2.

[4] Mabuchi F, Yoshimura K, Kashiwagi K, Yamagata Z, Kanba S, lijima H, et al. Risk factors for anxiety and depression in patients with glaucoma. Br J Ophthalmol. 2012;96:821-5.

[5] Zhou C, Qian S, Wu P, Qiu C. Anxiety and depression in Chinese patients with glaucoma: sociodemographic, clinical, and self-reported correlates. J Psychosom Res. 2013;75:75-82.

[6] Logean E, Dalimier E, Dainty C. Measured double-pass intensity point-spread function after adaptive optics correction of ocular aberrations. Opt Express. 2008;16:17348-57.

[7] Zhang Y, Zheng L, Bian A, Zhou Q. IOP measurement in silicone oil tamponade eyes by Corvis ST tonometer, Goldmann applanation tonometry and non-contact tonometry. Int Ophthalmol. 2018;38:697703.

[8] Wang YJ, Yang YN, Huang LY, Wang B, Han YC, Yan JB. Optical Quality and Related Factors in Ocular Hypertension: Preliminary Study. J Ophthalmol. 2016;2016:3071036.

[9] Liu L, Wang Y, Liu J, Liu W. Retinal-image quality and contrast sensitivity function in eyes with epiretinal membrane: a cross-sectional observational clinical study. BMC Ophthalmol. 2018;18:290.

[10] Lee K, Sohn J, Choi JG, Chung SK. Optical quality in central serous chorioretinopathy. Invest Ophthalmol Vis Sci. 2014;55:8598-603. 
[11] Hyman LG, Komaroff E, Heijl A, Bengtsson B, Leske MC, Early Manifest Glaucoma Trial G. Treatment and vision-related quality of life in the early manifest glaucoma trial. Ophthalmology. 2005;112:1505-13.

[12] Gedde SJ, Schiffman JC, Feuer WJ, Parrish RK, 2nd, Heuer DK, Brandt JD, et al. The tube versus trabeculectomy study: design and baseline characteristics of study patients. Am J Ophthalmol. $2005 ; 140: 275-87$.

[13] McKean-Cowdin R, Wang Y, Wu J, Azen SP, Varma R, Los Angeles Latino Eye Study G. Impact of visual field loss on health-related quality of life in glaucoma: the Los Angeles Latino Eye Study. Ophthalmology. 2008;115:941-8 e1.

[14] Gracitelli CP, Abe RY, Tatham AJ, Rosen PN, Zangwill LM, Boer ER, et al. Association between progressive retinal nerve fiber layer loss and longitudinal change in quality of life in glaucoma. JAMA Ophthalmol. 2015;133:384-90.

[15] Gutierrez P, Wilson MR, Johnson C, Gordon M, Cioffi GA, Ritch R, et al. Influence of glaucomatous visual field loss on health-related quality of life. Arch Ophthalmol. 1997;115:777-84.

[16] van Gestel A, Webers CA, Beckers HJ, van Dongen MC, Severens JL, Hendrikse F, et al. The relationship between visual field loss in glaucoma and health-related quality-of-life. Eye (Lond). 2010;24:1759-69.

[17] Lisboa R, Chun YS, Zangwill LM, Weinreb RN, Rosen PN, Liebmann JM, et al. Association between rates of binocular visual field loss and vision-related quality of life in patients with glaucoma. JAMA Ophthalmol. 2013;131:486-94.

[18] Jampel HD, Schwartz A, Pollack I, Abrams D, Weiss H, Miller R. Glaucoma patients' assessment of their visual function and quality of life. J Glaucoma. 2002;11:154-63.

\section{Tables}

Table 1 OQAS assessed optic quality, vision field, and the correlation coefficients between them in 64 POAG patients. The data were shown in mean \pm SD or median 


\begin{tabular}{|c|c|c|c|}
\hline & Better eye & Worse eye & Total \\
\hline MD & $5.50 \pm 5.71$ & $9.88 \pm 6.85$ & $7.69 \pm 6.65$ \\
\hline MTF cutoff & $25.52 \pm 14.58$ & $15.78 \pm 11.84$ & $20.64 \pm 14.10$ \\
& $(-0.593,<0.001)$ & $(-0.340,<0.006)$ & $(-0.551,<0.001)$ \\
\hline Mean OV & $0.83 \pm 0.48$ & $0.51 \pm 0.38$ & $0.67 \pm 0.47$ \\
& $(-0.446,<0.001)$ & $(-0.409,0.001)$ & $(-0.558,<0.001)$ \\
& $(0.320,0.010)$ & $(0.152,0.232)$ & $(0.152,0.087)$ \\
\hline OSI (median) & 1.15 & 2.75 & 2 \\
& & & \\
\hline
\end{tabular}

OQAS: Optical Quality Analysis System; OSI: objective scatter index; OV: OQAS values; POAG: rimary open angle glaucoma; MTF cutoff: modulation transfer function cut off frequency; MD: mean deviation

Bold characters indicate $P<0.05$

Table 2. $P$ values and correlation coefficients for single linear regression analysis of the association between visual function and visual disability indices of total participants 


\begin{tabular}{|c|c|c|c|c|c|c|c|c|}
\hline & \multicolumn{2}{|c|}{ MD } & \multicolumn{2}{|c|}{ MTF cutoff } & \multicolumn{2}{|c|}{ mean OV } & \multicolumn{2}{|c|}{ OSI } \\
\hline & Better eye & Worse eye & Better eye & Worse eye & Better eye & Worse eye & Better eye & Worse eye \\
\hline General health & $-0.042(0.744)$ & $0.049(0.699)$ & $0.209(0.097)$ & $0.405(0.001)$ & $0.258(0.039)$ & $0.426(<0.001)$ & $-0.122(0.338)$ & $0.016(0.899)$ \\
\hline General vision & $-0.423(0.001)$ & $-0.454(<0.001)$ & $0.310(0.013)$ & $0.451(<0.001)$ & $0.299(0.017)$ & $0.413(0.001)$ & $-0.104(0.414)$ & $-0.034(0.787)$ \\
\hline Ocular pain & $-0.023(0.858)$ & $0.107(0.402)$ & $0.041(0.749)$ & $0.074(0.562)$ & $0.083(0.513)$ & $0.103(0.418)$ & $0.084(0.509)$ & $0.161(0.205)$ \\
\hline Near vision & $-0.509(<0.001)$ & $-0.312(\mathbf{0 . 0 1 2})$ & $0.300(0.016)$ & $0.240(0.056)$ & $0.335(0.007)$ & $0.257(\mathbf{0 . 0 4 1 )}$ & $0.009(0.943)$ & $0.072(0.574)$ \\
\hline Distance vision & $-0.611(<0.001)$ & $-0.534(<0.001)$ & $0.351(0.004)$ & $0.273(0.029)$ & $0.362(0.003)$ & $0.270(0.031)$ & $-0.065(0.610)$ & $-0.024(0.851)$ \\
\hline Social function & $-0.166(0.189)$ & $-0.148(0.242)$ & $0.011(0.933)$ & $0.204(0.106)$ & $0.025(0.843)$ & $0.188(0.137)$ & $0.180(0.154)$ & $0.191(0.120)$ \\
\hline Mental Health & $-0.088(0.492)$ & $-0.099(0.436)$ & $0.082(0.520)$ & $0.200(0.113)$ & $0.067(0.598)$ & $0.179(0.156)$ & $0.118(0.353)$ & $0.162(0.202)$ \\
\hline Role limitations & $-0.550(<0.001)$ & $-0.392(0.001)$ & $0.219(0.082)$ & $0.155(0.222)$ & $0.227(0.071)$ & $0.136(0.284)$ & $0.067(0.597)$ & $0.058(0.646)$ \\
\hline Dependency & $-0.184(0.145)$ & $-0.310(\mathbf{0 . 0 1 3})$ & $0.249(0.047)$ & $0.345(0.005)$ & $0.206(0.103)$ & $0.325(0.009)$ & $-0.054(0.669)$ & $0.063(0.620)$ \\
\hline Driving & $-0.553(0.002)$ & $-0.358(0.045)$ & $0.289(0.122)$ & $-0.002(0.993)$ & $0.327(\mathbf{0 . 0 7 8})$ & $0.077(0.684)$ & $-0.146(0.441)$ & $-0.172(0.364)$ \\
\hline Color vision & $-0.386(0.002)$ & $0.214(0.090)$ & $0.060(0.636)$ & $0.086(0.500)$ & $0.108(0.398)$ & $0.086(0.499)$ & $0.225(0.074)$ & $0.173(0.172)$ \\
\hline Peripheral vision & $-0.516(<0.001)$ & $-0.617(<0.001)$ & $0.410(0.001)$ & $0.439(<0.001)$ & $0.406(0.001)$ & $0.411(0.001)$ & $-0.217(0.084)$ & $-0.198(0.116)$ \\
\hline Composite & $-0.426(<0.001)$ & $-0.278(0.026)$ & $0.213(0.091)$ & $0.262(0.037)$ & $0.218(0.083)$ & $0.253(0.044)$ & $0.084(0.510)$ & $0.117(0.356)$ \\
\hline
\end{tabular}

OSI: objective scatter index; OV: OQAS values; MTF cutoff: modulation transfer function cut off frequency; MD: mean deviation

Bold characters indicate $P<0.05$

Figures 


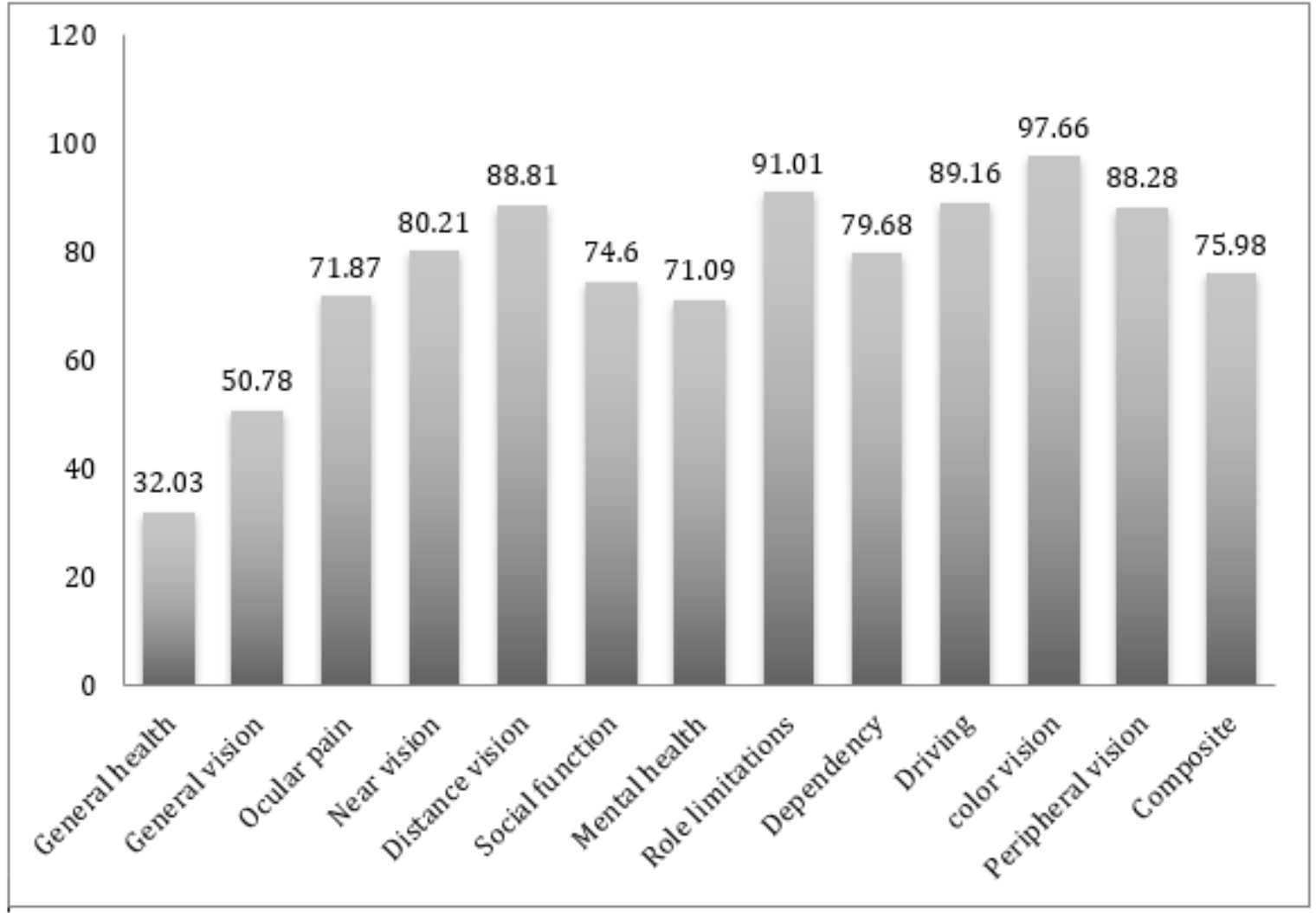

Figure 1

The subscale scores for primary open angle glaucoma patients on a Chinese version of the NEI VFQ-25

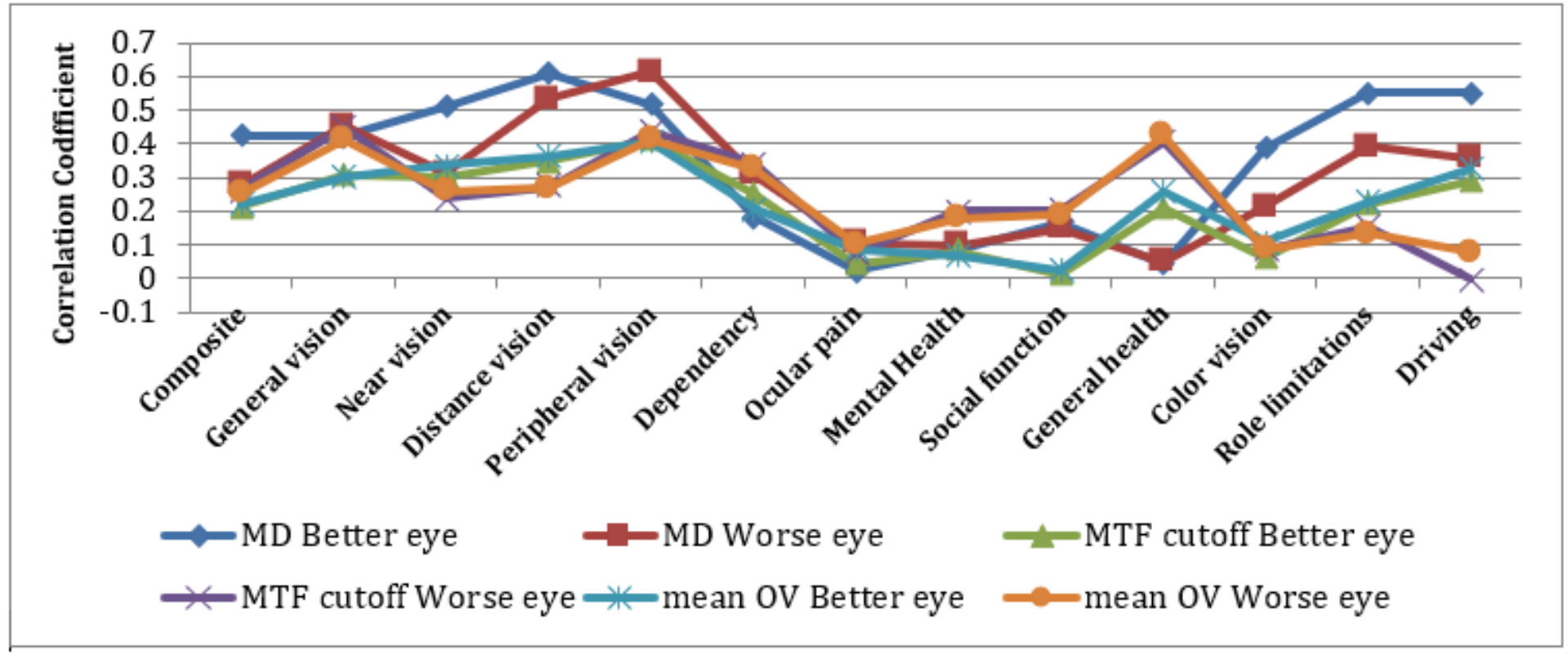

Figure 2

The correlation coefficients between mean deviation (MD), modulation transfer function cut off frequency (MTF cutoff), mean OQAS values (OV) and quality of life (QoL) 